\title{
Integral Transforms on a Function Space with Change of Scales Using Multivariate Normal Distributions
}

\author{
Dong Hyun Cho \\ Department of Mathematics, Kyonggi University, Suwon 16227, Republic of Korea \\ Correspondence should be addressed to Dong Hyun Cho; j94385@kyonggi.ac.kr
}

Received 2 March 2016; Accepted 4 May 2016

Academic Editor: Alberto Fiorenza

Copyright (c) 2016 Dong Hyun Cho. This is an open access article distributed under the Creative Commons Attribution License, which permits unrestricted use, distribution, and reproduction in any medium, provided the original work is properly cited.

\begin{abstract}
Using simple formulas for generalized conditional Wiener integrals on a function space which is an analogue of Wiener space, we evaluate two generalized analytic conditional Wiener integrals of a generalized cylinder function which is useful in Feynman integration theories and quantum mechanics. We then establish various integral transforms over continuous paths with change of scales for the generalized analytic conditional Wiener integrals. In these evaluation formulas and integral transforms we use multivariate normal distributions so that the orthonormalization process of projection vectors which are needed to establish the conditional Wiener integrals can be removed in the existing change of scale transforms. Consequently the transforms in the present paper can be expressed in terms of the generalized cylinder function itself.
\end{abstract}

\section{Introduction}

Let $C_{0}[0, T]$ denote the classical Wiener space, the space of continuous real-valued functions $x$ on $[0, T]$ with $x(0)=0$. It is well known that Wiener measure and Wiener measurability are not invariant under change of scale and under translation $[1,2]$. As integral transforms, change of scale formulas for Wiener integrals of various functions was developed on the classical and abstract Wiener spaces [3-7]. Further change of scale formulas for conditional Wiener integrals was introduced by the author and his coauthors [8-10]. In fact change of scale formulas for conditional Wiener integrals was established on $C_{0}[0, T]$, on the infinite dimensional Wiener space, and on $C[0, T]$, an analogue of Wiener space [11] which is the space of real-valued continuous paths on $[0, T]$. Some difficulties in studying the transforms for the conditional Wiener integrals of cylinder functions which play important roles in Feynman integration theories are that they cannot be expressed in terms of the original cylinder functions. To avoid these difficulties, modified cylinder functions expressed by a polygonal function with projection vectors satisfying orthonormality were used to derive the change of scale transforms [8-10]. In this paper we use multivariate normal distributions so that the orthonormalization process of projection vectors which are needed to establish the conditional Wiener integrals can be removed in the existing change of scale transforms. Consequently the transforms in the present paper can be expressed by the cylinder function itself and generalize the results in [8-10].

Let $a \in C[0, T]$ and let $h$ be of bounded variation with $h \neq 0$ a.e. on $[0, T]$. Define a stochastic process $Z: C[0, T] \times$ $[0, T] \rightarrow \mathbb{R}$ by

$$
Z(x, t)=\int_{0}^{t} h(s) d x(s)+x(0)+a(t)
$$

for $x \in C[0, T]$ and $t \in[0, T]$, where the integral denotes a generalized Paley-Wiener-Zygmund stochastic integral. For a partition $0=t_{0}<t_{1}<\cdots<t_{n}<t_{n+1}=T$ of $[0, T]$ define random vectors $Z_{n}: C[0, T] \rightarrow \mathbb{R}^{n+1}$ and $Z_{n+1}: C[0, T] \rightarrow$ $\mathbb{R}^{n+2}$ by

$$
\begin{aligned}
& Z_{n}(x)=\left(Z\left(x, t_{0}\right), Z\left(x, t_{1}\right), \ldots, Z\left(x, t_{n}\right)\right), \\
& Z_{n+1}(x) \\
& \quad=\left(Z\left(x, t_{0}\right), Z\left(x, t_{1}\right), \ldots, Z\left(x, t_{n}\right), Z\left(x, t_{n+1}\right)\right) .
\end{aligned}
$$

Using simple formulas for generalized conditional Wiener integrals on $C[0, T]$ with the conditioning functions $Z_{n}$ and 
$Z_{n+1}[12]$ we evaluate generalized analytic conditional Wiener integrals of the following generalized cylinder function:

$$
\begin{aligned}
F_{Z} & (x) \\
& =f\left(\int_{0}^{T} v_{1}(s) d Z(x, s), \ldots, \int_{0}^{T} v_{r}(s) d Z(x, s)\right),
\end{aligned}
$$

where $f \in L_{p}\left(\mathbb{R}^{r}\right)$ with $1 \leq p \leq \infty$ and $\left\{v_{1}, \ldots, v_{r}\right\}$ is an orthonormal subset of $L_{2}[0, T]$. We then establish various change of scale transforms for the generalized analytic conditional Wiener integrals of $F_{Z}$ with $Z_{n}$ and $Z_{n+1}$. In these evaluation formulas and scale transforms we use multivariate normal distributions so that Gram-Schmidt orthonormalization process of $\left\{\mathscr{P}^{\perp} h v_{1}, \ldots, \mathscr{P}^{\perp} h v_{r}\right\}$ can be removed in the existing change of scale transforms for a suitable orthogonal projection $\mathscr{P}^{\perp}$ on $L_{2}[0, T]$. In contrast with the existing change of scale transforms in [8-10], the transforms in this paper are expressed in terms of the cylinder function $F_{Z}$ itself and generalize some results in those references.

\section{An Analogue of Wiener Space and Preliminary Results}

In this section we will introduce the analogue of Wiener space $C[0, T]$ and the preliminary results which are needed in the following sections.

For a positive real $T$ let $C[0, T]$ denote the space of realvalued continuous functions on the time interval $[0, T]$ with the supremum norm. For $\vec{t}=\left(t_{0}, t_{1}, \ldots, t_{n}\right)$ with $0=t_{0}<t_{1}<$ $\cdots<t_{n} \leq T$ let $J_{\vec{t}}: C[0, T] \rightarrow \mathbb{R}^{n+1}$ be the function given by

$$
J_{\vec{t}}(x)=\left(x\left(t_{0}\right), x\left(t_{1}\right), \ldots, x\left(t_{n}\right)\right) .
$$

For $B_{j}(j=0,1, \ldots, n)$ in $\mathscr{B}(\mathbb{R})$, the subset $J_{\vec{t}}^{-1}\left(\prod_{j=0}^{n} B_{j}\right)$ of $C[0, T]$ is called an interval and let $\mathscr{I}$ be the set of all such intervals. For a probability measure $\varphi$ on $\mathscr{B}(\mathbb{R})$ let

$$
\begin{gathered}
m_{\varphi}\left[J_{\vec{t}}^{-1}\left(\prod_{j=0}^{n} B_{j}\right)\right]=\left[\prod_{j=1}^{n} \frac{1}{2 \pi\left(t_{j}-t_{j-1}\right)}\right]^{1 / 2} \\
\cdot \int_{B_{0}} \int_{\prod_{j=1}^{n} B_{j}} W_{n}\left(\vec{t}, \vec{u}, u_{0}\right) d \vec{u} d \varphi\left(u_{0}\right),
\end{gathered}
$$

where for $\vec{t}=\left(t_{0}, t_{1}, \ldots, t_{n}\right)$ and $\vec{u}=\left(u_{1}, \ldots, u_{n}\right)$

$$
W_{n}\left(\vec{t}, \vec{u}, u_{0}\right)=\exp \left\{-\frac{1}{2} \sum_{j=1}^{n} \frac{\left(u_{j}-u_{j-1}\right)^{2}}{t_{j}-t_{j-1}}\right\} .
$$

$\mathscr{B}(C[0, T])$, the Borel $\sigma$-algebra of $C[0, T]$, coincides with the smallest $\sigma$-algebra generated by $\mathscr{I}$ and there exists a unique probability measure $w_{\varphi}$ on $C[0, T]$ such that $w_{\varphi}(I)=m_{\varphi}(I)$ for all $I \in \mathscr{I}$. This measure $w_{\varphi}$ is called an analogue of Wiener measure associated with the probability measure $\varphi$ [11]. Let $\left\{e_{k}: k=1,2, \ldots\right\}$ be a complete orthonormal subset of $L_{2}[0, T]$ such that each $e_{k}$ is of bounded variation. For $v \in L_{2}[0, T]$ and $x$ in $C[0, T]$ let

$$
(v, x)=\lim _{n \rightarrow \infty} \sum_{k=1}^{n} \int_{0}^{T}\left\langle v, e_{k}\right\rangle e_{k}(t) d x(t)
$$

if the limit exists, where $\langle\cdot, \cdot\rangle$ denotes the inner product over $L_{2}[0, T] .(v, x)$ is called the Paley-Wiener-Zygmund integral of $v$ associated with $x$.

Let $\mathbb{C}$ and $\mathbb{C}_{+}$denote the sets of complex numbers and complex numbers with positive real parts, respectively. Let $F: C[0, T] \rightarrow \mathbb{C}$ be integrable and let $X$ be a random vector on $C[0, T]$ assuming that the value space of $X$ is a normed space with the Borel $\sigma$-algebra. Then we have the conditional expectation $E[F \mid X]$ of $F$ given $X$ from a well-known probability theory. Furthermore there exists a $P_{X}$-integrable complex valued function $\psi$ on the value space of $X$ such that $E[F \mid X](x)=(\psi \circ X)(x)$ for $w_{\varphi}$ a.e. $x \in C[0, T]$, where $P_{X}$ is the probability distribution of $X$. The function $\psi$ is called the conditional $w_{\varphi}$-integral of $F$ given $X$ and it is also denoted by $E[F \mid X]$.

Let $0=t_{0}<t_{1}<\cdots<t_{n}<t_{n+1}=T$ be a partition of $[0, T]$, where $n$ is a fixed nonnegative integer. Let $h \in L_{2}[0, T]$ be of bounded variation with $h \neq 0$ a.e. on $[0, T]$. For $j=$ $1, \ldots, n, n+1$ let

$$
\alpha_{j}=\frac{1}{\left\|\chi_{\left(t_{j-1}, t_{j}\right]} h\right\|} \chi_{\left(t_{j-1}, t_{j}\right]} h,
$$

let $V$ be the subspace of $L_{2}[0, T]$ generated by $\left\{\alpha_{1}, \ldots\right.$, $\left.\alpha_{n}, \alpha_{n+1}\right\}$, and let $V^{\perp}$ be the orthogonal complement of $V$. Let $\mathscr{P}: L_{2}[0, T] \rightarrow V$ be the orthogonal projection given by $\mathscr{P} v=\sum_{j=1}^{n+1}\left\langle v, \alpha_{j}\right\rangle \alpha_{j}$ and let $\mathscr{P}^{\perp}: L_{2}[0, T] \rightarrow V^{\perp}$ be an orthogonal projection. Let $a \in C[0, T]$ and let it be absolutely continuous. Define stochastic processes $X, Z: C[0, T] \times$ $[0, T] \rightarrow \mathbb{R}$ by

$$
\begin{aligned}
& X(x, t)=\left(h \chi_{[0, t]}, x\right) \\
& Z(x, t)=\left(h \chi_{[0, t]}, x\right)+x(0)+a(t)
\end{aligned}
$$

for $x \in C[0, T]$ and $t \in[0, T]$. Define random vectors $Z_{n}$ : $C[0, T] \rightarrow \mathbb{R}^{n+1}$ and $Z_{n+1}: C[0, T] \rightarrow \mathbb{R}^{n+2}$ by

$$
\begin{aligned}
& Z_{n}(x)=\left(Z\left(x, t_{0}\right), Z\left(x, t_{1}\right), \ldots, Z\left(x, t_{n}\right)\right), \\
& Z_{n+1}(x) \\
& \quad=\left(Z\left(x, t_{0}\right), Z\left(x, t_{1}\right), \ldots, Z\left(x, t_{n}\right), Z\left(x, t_{n+1}\right)\right)
\end{aligned}
$$

for $x \in C[0, T]$. For $t \in[0, T]$ let $b(t)=\int_{0}^{t}[h(s)]^{2} d s$ and for any function $f$ on $[0, T]$ define the polygonal function $P_{b, n+1}(f)$ of $f$ by

$$
\begin{aligned}
& P_{b, n+1}(f)(t)=\sum_{j=1}^{n+1}\left[\frac{b\left(t_{j}\right)-b(t)}{b\left(t_{j}\right)-b\left(t_{j-1}\right)} f\left(t_{j-1}\right)\right. \\
& \left.+\frac{b(t)-b\left(t_{j-1}\right)}{b\left(t_{j}\right)-b\left(t_{j-1}\right)} f\left(t_{j}\right)\right] \chi_{\left(t_{j-1}, t_{j}\right]}(t)+f(0) \chi_{\{0\}}(t)
\end{aligned}
$$

for $t \in[0, T]$, where $\chi_{\left(t_{j-1}, t_{j}\right]}$ and $\chi_{\{0\}}$ denote the indicator functions on the interval $[0, T]$. For $\vec{\xi}_{n+1}=\left(\xi_{0}, \xi_{1}, \ldots, \xi_{n}\right.$, $\left.\xi_{n+1}\right) \in \mathbb{R}^{n+2}$ define the polygonal function $P_{b, n+1}\left(\vec{\xi}_{n+1}\right)$ of $\vec{\xi}_{n+1}$ by (11) with $f\left(t_{j}\right)$ replaced by $\xi_{j}$ for $j=0,1, \ldots, n, n+1$. 
If $\vec{\xi}_{n}=\left(\xi_{0}, \xi_{1}, \ldots, \xi_{n}\right) \in \mathbb{R}^{n+1}, P_{b, n}\left(\vec{\xi}_{n}\right)$ is interpreted as $P_{b, n+1}\left(\vec{\xi}_{n+1}\right) \chi_{\left[0, t_{n}\right]}$ on $[0, T]$. For $x \in C[0, T]$ and $t \in[0, T]$ let

$$
\begin{aligned}
A(t) & =a(t)-P_{b, n+1}(a)(t), \\
X_{b, n+1}(x, t) & =X(x, t)-P_{b, n+1}(X(x, \cdot))(t), \\
Z_{b, n+1}(x, t) & =Z(x, t)-P_{b, n+1}(Z(x, \cdot))(t) .
\end{aligned}
$$

For $\vec{a}, \vec{u} \in \mathbb{R}^{r}, \lambda \in \mathbb{C}$, and any nonsingular positive $r \times r$ matrix $A_{r}$ on $\mathbb{R}$ let

$$
\begin{aligned}
& \Psi_{r}\left(\lambda, \vec{a}, A_{r}, \vec{u}\right)=\left[\frac{\lambda^{r}}{(2 \pi)^{r}\left|A_{r}\right|}\right]^{1 / 2} \\
& \cdot \exp \left\{-\frac{\lambda}{2}\left\langle A_{r}^{-1}(\vec{u}-\vec{a}), \vec{u}-\vec{a}\right\rangle_{\mathbb{R}}\right\},
\end{aligned}
$$

where $\langle\cdot, \cdot\rangle_{\mathbb{R}}$ denotes the dot product on $\mathbb{R}^{r}$. For a function $F: C[0, T] \rightarrow \mathbb{C}$ let $F_{Z}(x)=F(Z(x, \cdot))$ for $x \in C[0, T]$. By Theorems 6 and 7 in [12], we have the following theorems.

Theorem 1. Let $F$ be a complex valued function on $C[0, T]$ and let $F_{Z}$ be integrable over $C[0, T]$. Then for $P_{Z_{n+1}}$ a.e. $\vec{\xi}_{n+1} \in \mathbb{R}^{n+2}$

$$
\begin{aligned}
& E\left[F_{Z} \mid Z_{n+1}\right]\left(\vec{\xi}_{n+1}\right) \\
& \quad=E\left[F\left(Z_{b, n+1}(x, \cdot)+P_{b, n+1}\left(\vec{\xi}_{n+1}\right)\right)\right],
\end{aligned}
$$

where $Z_{b, n+1}$ is given by (14), $P_{Z_{n+1}}$ is the probability distribution of $Z_{n+1}$ on $\left(\mathbb{R}^{n+2}, B\left(\mathbb{R}^{n+2}\right)\right)$, and the expectation on the right hand side of the equation is taken over the variable $x$.

Theorem 2. Let $F_{Z}$ be integrable over $C[0, T]$ and let $P_{Z_{n}}$ be the probability distribution of $Z_{n}$ on $\left(\mathbb{R}^{n+1}, \mathscr{B}\left(\mathbb{R}^{n+1}\right)\right)$. Then for $P_{Z_{n}}$ a.e. $\vec{\xi}_{n}=\left(\xi_{0}, \xi_{1}, \ldots, \xi_{n}\right) \in \mathbb{R}^{n+1}$

$$
\begin{aligned}
E & {\left[F_{Z} \mid Z_{n}\right]\left(\vec{\xi}_{n}\right)=\int_{\mathbb{R}} \Psi_{1}\left(1, a(T)-a\left(t_{n}\right), b(T)\right.} \\
& \left.-b\left(t_{n}\right), \xi_{n+1}-\xi_{n}\right) \\
& \cdot E\left[F\left(Z_{b, n+1}(x, \cdot)+P_{b, n+1}\left(\vec{\xi}_{n+1}\right)\right)\right] d \xi_{n+1},
\end{aligned}
$$

where $\vec{\xi}_{n+1}=\left(\xi_{0}, \xi_{1}, \ldots, \xi_{n}, \xi_{n+1}\right)$ and $\Psi_{1}$ is given by (15) with $r=1$.

For $\lambda>0$ and $x \in C[0, T]$ let $F_{Z}^{\lambda}(x)=F_{Z}\left(\lambda^{-1 / 2} x\right)$, $Z_{n}^{\lambda}(x)=Z_{n}\left(\lambda^{-1 / 2} x\right)$, and $Z_{n+1}^{\lambda}(x)=Z_{n+1}\left(\lambda^{-1 / 2} x\right)$. Suppose that $E\left[F_{Z}^{\lambda}\right]$ exists. By Theorem 1

$$
\begin{aligned}
E & {\left[F_{Z}^{\lambda} \mid Z_{n+1}^{\lambda}\right]\left(\vec{\xi}_{n+1}\right) } \\
& =E\left[F\left(\lambda^{-1 / 2} X_{b, n+1}(x, \cdot)+A+P_{b, n+1}\left(\vec{\xi}_{n+1}\right)\right)\right]
\end{aligned}
$$

for $P_{Z_{n+1}^{\lambda}}$ a.e. $\vec{\xi}_{n+1} \in \mathbb{R}^{n+2}$, where $A$ and $X_{b, n+1}$ are given by (12) and (13), respectively, and $P_{Z_{n+1}^{\lambda}}$ is the probability distribution of $Z_{n+1}^{\lambda}$ on $\left(\mathbb{R}^{n+2}, \mathscr{B}\left(\mathbb{R}^{n+2}\right)\right)$. By Theorem 2 we also have for $P_{Z_{n}^{\lambda}}$ a.e. $\vec{\xi}_{n}=\left(\xi_{0}, \xi_{1}, \ldots, \xi_{n}\right) \in \mathbb{R}^{n+1}$

$$
\begin{aligned}
& E\left[F_{Z}^{\lambda} \mid Z_{n}^{\lambda}\right]\left(\vec{\xi}_{n}\right)=\int_{\mathbb{R}} \Psi_{1}\left(\lambda, a(T)-a\left(t_{n}\right), b(T)\right. \\
& \left.\quad-b\left(t_{n}\right), \xi_{n+1}-\xi_{n}\right) E\left[F \left(\lambda^{-1 / 2} X_{b, n+1}(x, \cdot)+A\right.\right. \\
& \left.\left.\quad+P_{b, n+1}\left(\vec{\xi}_{n+1}\right)\right)\right] d \xi_{n+1},
\end{aligned}
$$

where $\vec{\xi}_{n+1}=\left(\xi_{0}, \xi_{1}, \ldots, \xi_{n}, \xi_{n+1}\right)$ and $P_{Z_{n}^{\lambda}}$ is the probability distribution of $Z_{n}^{\lambda}$ on $\left(\mathbb{R}^{n+1}, \mathscr{B}\left(\mathbb{R}^{n+1}\right)\right)$.

Let $I_{F_{Z}}^{\lambda}\left(\vec{\xi}_{n+1}\right)$ and $K_{F_{Z}}^{\lambda}\left(\vec{\xi}_{n}\right)$ be the right hand sides of (18) and (19), respectively. If $I_{F_{Z}}^{\lambda}\left(\vec{\xi}_{n+1}\right)$ has an analytic extension $J_{\lambda}^{*}\left(F_{Z}\right)\left(\vec{\xi}_{n+1}\right)$ on $\mathbb{C}_{+}$, then it is called the conditional analytic Wiener $w_{\varphi}$-integral of $F_{Z}$ given $Z_{n+1}$ with the parameter $\lambda$ and is denoted by

$$
E^{a n w_{\lambda}}\left[F_{Z} \mid Z_{n+1}\right]\left(\vec{\xi}_{n+1}\right)=J_{\lambda}^{*}\left(F_{Z}\right)\left(\vec{\xi}_{n+1}\right)
$$

for $\vec{\xi}_{n+1} \in \mathbb{R}^{n+2}$. Moreover if, for nonzero real $q$, $E^{a n w_{\lambda}}\left[F_{Z} \mid\right.$ $\left.Z_{n+1}\right]\left(\vec{\xi}_{n+1}\right)$ has a limit as $\lambda$ approaches to $-i q$ through $\mathbb{C}_{+}$, then it is called the conditional analytic Feynman $w_{\varphi}$-integral of $F_{Z}$ given $Z_{n+1}$ with the parameter $q$ and is denoted by

$$
\begin{aligned}
& E^{a n f_{q}}\left[F_{Z} \mid Z_{n+1}\right]\left(\vec{\xi}_{n+1}\right) \\
& \quad=\lim _{\lambda \rightarrow-i q} E^{a n w_{\lambda}}\left[F_{Z} \mid Z_{n+1}\right]\left(\vec{\xi}_{n+1}\right) .
\end{aligned}
$$

Replacing $I_{F_{Z}}^{\lambda}\left(\vec{\xi}_{n+1}\right)$ by $K_{F_{Z}}^{\lambda}\left(\vec{\xi}_{n}\right)$, we define $E^{a n w_{\lambda}}\left[F_{Z} \mid Z_{n}\right]\left(\vec{\xi}_{n}\right)$ and $E^{a n f_{a}}\left[F_{Z} \mid Z_{n}\right]\left(\vec{\xi}_{n}\right)$. If $E\left[F_{Z}^{\lambda}\right]$ exists for $\lambda>0$ and it has an analytic extension $J_{\lambda}^{*}\left(F_{Z}\right)$ on $\mathbb{C}_{+}$, then we call $J_{\lambda}^{*}\left(F_{Z}\right)$ the analytic Wiener $w_{\varphi}$-integral of $F$ over $C[0, T]$ with parameter $\lambda$ and it is denoted by

$$
E^{a n w_{\lambda}}\left[F_{Z}\right]=J_{\lambda}^{*}\left(F_{Z}\right) .
$$

$E^{a n f_{q}}\left[F_{Z}\right]$ is also defined by

$$
E^{a n f_{q}}\left[F_{Z}\right]=\lim _{\lambda \rightarrow-i q} E^{a n w_{\lambda}}\left[F_{Z}\right],
$$

if it exists, where the limit is taken through $\mathbb{C}_{+}$.

Applying Theorem 2.3 in [13], we can easily prove the following theorem.

Theorem 3. Let $\left\{h_{1}, h_{2}, \ldots, h_{r}\right\}$ be an orthonormal system of $L_{2}[0, T]$. Then $\left(h_{1}, \cdot\right), \ldots,\left(h_{r}, \cdot\right)$ are independent and each $\left(h_{i}, \cdot\right)$ has the standard normal distribution. Moreover if $f$ : $\mathbb{R}^{r+1} \rightarrow \mathbb{R}$ is Borel measurable, then

$$
\begin{aligned}
& \int_{C[0, T]} f\left(x(0),\left(h_{1}, x\right), \ldots,\left(h_{r}, x\right)\right) d w_{\varphi}(x) \\
& \stackrel{*}{=} \int_{\mathbb{R}} \int_{\mathbb{R}^{r}} f\left(u_{0}, \vec{u}\right) \Psi_{r}\left(1, \overrightarrow{0}, I_{r}, \vec{u}\right) d \vec{u} d \varphi\left(u_{0}\right),
\end{aligned}
$$

where $I_{r}$ is the identity matrix on $\mathbb{R}^{r}$ and $\stackrel{*}{=}$ means that if either side exists then both sides exist and they are equal. 
Since $\varphi$ is a probability measure on $\mathbb{R}$ we have the following corollary.

Corollary 4. Under the assumptions as given in Theorem 3

$$
\begin{gathered}
\int_{C[0, T]} f\left(\left(h_{1}, x\right), \ldots,\left(h_{r}, x\right)\right) d w_{\varphi}(x) \\
\stackrel{*}{=} \int_{\mathbb{R}^{r}} f(\vec{u}) \Psi_{r}\left(1, \overrightarrow{0}, I_{r}, \vec{u}\right) d \vec{u}
\end{gathered}
$$

if $f: \mathbb{R}^{r} \rightarrow \mathbb{R}$ is Borel measurable.

The following lemmas are useful to prove the results in the next sections and their proofs are simple.

Lemma 5. Let $v \in L_{2}[0, T]$. Then for $w_{\varphi}$ a.e. $x \in C[0, T]$

$$
\left(v, P_{b, n+1}(X(x, \cdot))\right)=\left(\mathscr{P} M_{h} v, x\right),
$$

where $M_{h}: L_{2}[0, T] \rightarrow L_{2}[0, T]$ is the multiplication operator defined by

$$
M_{h} u=h u, \quad \text { for } u \in L_{2}[0, T] \text {. }
$$

Lemma 6. Let $v \in L_{2}[0, T], \vec{\xi}_{n+1}=\left(\xi_{0}, \xi_{1}, \ldots, \xi_{n}, \xi_{n+1}\right) \in$ $\mathbb{R}^{n+2}$, and

$$
\left(v, P_{b, n}\left(\vec{\xi}_{n}\right)\right)=\sum_{j=1}^{n}\left\langle v \alpha_{j}, \alpha_{j}\right\rangle\left(\xi_{j}-\xi_{j-1}\right),
$$

where $\vec{\xi}_{n}=\left(\xi_{0}, \xi_{1}, \ldots, \xi_{n}\right)$. Then

$$
\begin{aligned}
\left(v, P_{b, n+1}\left(\vec{\xi}_{n+1}\right)\right)= & \sum_{j=1}^{n+1}\left\langle v \alpha_{j}, \alpha_{j}\right\rangle\left(\xi_{j}-\xi_{j-1}\right) \\
= & \left(v, P_{b, n}\left(\vec{\xi}_{n}\right)\right) \\
& +\left\langle v \alpha_{n+1}, \alpha_{n+1}\right\rangle\left(\xi_{n+1}-\xi_{n}\right) .
\end{aligned}
$$

Remark 7. (1) The multiplication operator $M_{h}$ in Lemma 5 is well defined because $h$ is of bounded variation which implies the boundedness of $h . M_{h}$ will denote the operator as given in the lemma unless otherwise specified.

(2) For $\vec{\xi}_{n}=\left(\xi_{0}, \xi_{1}, \ldots, \xi_{n}\right) \in \mathbb{R}^{n+1}$ it is possible that $P_{b, n}\left(\vec{\xi}_{n}\right) \notin C[0, T]$ if $\xi_{n} \neq 0$. In this case the symbol $\left(v, P_{b, n}\left(\vec{\xi}_{n}\right)\right)$ does not mean the Paley-Wiener-Zygmund integral of $v \in$ $L_{2}[0, T]$. It is only a formal expression for $\sum_{j=1}^{n}\left\langle v \alpha_{j}, \alpha_{j}\right\rangle\left(\xi_{j}-\right.$ $\left.\xi_{j-1}\right)$ which is as given in Lemma 6.

\section{Multivariate Normal Distributions}

In this section we derive a multivariate normal distribution which will be needed in the next section.

Lemma 8. Let $\left\{v_{1}, \ldots, v_{r}\right\}$ be a set of independent vectors in $L_{2}[0, T]$. Then the covariance matrix $\Sigma=\left[a_{i j}\right]_{r \times r}$ of the random variables $\left(v_{l}, \cdot\right), l=1, \ldots, r$, exists and is positive definite. Moreover $a_{i j}$ is given by

$$
a_{i j}=\left\langle v_{i}, v_{j}\right\rangle
$$

and the determinant $|\Sigma|$ of $\Sigma$ is positive so that $\Sigma$ is nonsingular and the inverse matrix $\Sigma^{-1}$ of $\sum$ is also positive definite.

Proof. By Theorem 3

$$
\begin{aligned}
& \left\|v_{i}\right\|^{2}+2 \int_{C[0, T]}\left(v_{i}, x\right)\left(v_{j}, x\right) d w_{\varphi}(x)+\left\|v_{j}\right\|^{2} \\
& =\int_{C[0, T]}\left(v_{i}+v_{j}, x\right)^{2} d w_{\varphi}(x) \\
& =\left\|v_{i}\right\|^{2}+2\left\langle v_{i}, v_{j}\right\rangle+\left\|v_{j}\right\|^{2}
\end{aligned}
$$

so that the covariance $a_{i j}$ of $\left(v_{i}, \cdot\right)$ and $\left(v_{j}, \cdot\right)$ is given by $a_{i j}=$ $\left\langle v_{i}, v_{j}\right\rangle$ which proves (30). We have for $\vec{c}=\left(c_{1}, \ldots, c_{r}\right) \in \mathbb{R}^{r}$

$$
\left\langle\sum \vec{c}, \vec{c}\right\rangle_{\mathbb{R}}=\sum_{l=1}^{r} \sum_{j=1}^{r} a_{l j} c_{l} c_{j}=\left\|\sum_{j=1}^{r} c_{j} v_{j}\right\|^{2} \geq 0 .
$$

Moreover if $\left\langle\sum \vec{c}, \vec{c}\right\rangle_{\mathbb{R}}=0$, then $\sum_{j=1}^{r} c_{j} v_{j}=0$ which implies $c_{j}=0$ for $j=1, \ldots, r$ by the assumption. Thus the covariance matrix $\Sigma$ is positive definite. Since $\Sigma$ is symmetric and positive definite, the eigenvalues $\lambda_{1}, \ldots, \lambda_{r}$ of $\Sigma$ are real and positive. Since $|\Sigma|=\prod_{j=1}^{r} \lambda_{j}>0, \Sigma$ is invertible. Since

$$
\left\langle\Sigma^{-1} \vec{c}, \vec{c}\right\rangle_{\mathbb{R}}=\left\langle\vec{c}, \Sigma^{-1} \vec{c}\right\rangle_{\mathbb{R}}=\left\langle\Sigma \Sigma^{-1} \vec{c}, \Sigma^{-1} \vec{c}\right\rangle_{\mathbb{R}} \geq 0
$$

$\left\langle\Sigma_{r}^{-1} \vec{c}, \vec{c}\right\rangle_{\mathbb{R}}=0$ implies $\Sigma_{r}^{-1} \vec{c}=\overrightarrow{0}$; that is, $\vec{c}=\overrightarrow{0}$ and $\Sigma^{-1}$ is positive definite.

For simplicity let

$$
(\vec{v}, x)=\left(\left(v_{1}, x\right), \ldots,\left(v_{r}, x\right)\right)
$$

for $x \in C[0, T]$ and $\left\{v_{1}, \ldots, v_{r}\right\} \subseteq L_{2}[0, T]$.

By Lemma 8 , Theorem 4 of [14], and the change of variable theorem, we have the following theorem.

Theorem 9. Let the assumptions and notations be as given in Lemma 8. Then for every Borel measurable function $f$ : $\mathbb{R}^{r+1} \rightarrow \mathbb{C}$

$$
\begin{aligned}
& \int_{C[0, T]} f(x(0),(\vec{v}, x)) d w_{\varphi}(x) \\
& \stackrel{*}{=} \int_{\mathbb{R}} \int_{\mathbb{R}^{r}} f\left(u_{0}, \vec{u}\right) \Psi_{r}(1, \overrightarrow{0}, \Sigma, \vec{u}) d \vec{u} d \varphi\left(u_{0}\right) \\
& \quad \stackrel{*}{=} \int_{\mathbb{R}} \int_{\mathbb{R}^{r}} f\left(u_{0}, \Sigma^{1 / 2} \vec{u}\right) \Psi_{r}\left(1, \overrightarrow{0}, I_{r}, \vec{u}\right) d \vec{u} d \varphi\left(u_{0}\right),
\end{aligned}
$$

where $\Psi_{r}$ is given by (15), $I_{r}$ is the identity matrix on $\mathbb{R}$, and $\Sigma^{1 / 2}$ is the positive definite matrix satisfying $\left(\Sigma^{1 / 2}\right)^{2}=\Sigma$.

By the same process as used in Lemma 2.1 of [15], Theorem 9, and the change of variable theorem, we have the following corollary. 
Corollary 10. Let $\left\{v_{1}, \ldots, v_{r}\right\}$ be a subset of $L_{2}[0, T]$ and suppose that $\left\{M_{h} v_{1}, \ldots, M_{h} v_{r}\right\}$ is an independent set. Then the random vector $(\vec{v}, Z(x, \cdot))$ has the multivariate normal distribution with mean vector $(\vec{v}, a)$ and covariance matrix $\Sigma_{M_{h}}=\left[\left\langle M_{h} v_{i}, M_{h} v_{j}\right\rangle\right]_{r \times r}$. Moreover, for any Borel measurable function $f: \mathbb{R}^{r+1} \rightarrow \mathbb{C}$, we have

$$
\begin{aligned}
& \int_{C[0, T]} f(x(0),(\vec{v}, Z(x, \cdot))) d w_{\varphi}(x) \\
& \stackrel{*}{=} \int_{\mathbb{R}} \int_{\mathbb{R}^{r}} f\left(u_{0}, \vec{u}\right) \\
& \cdot \Psi_{r}\left(1,(\vec{v}, a), \Sigma_{M_{h}}, \vec{u}\right) d \vec{u} d \varphi\left(u_{0}\right) \\
& \quad * \int_{\mathbb{R}} \int_{\mathbb{R}^{r}} f\left(u_{0}, \Sigma_{M_{h}}^{1 / 2} \vec{u}+(\vec{v}, a)\right) \\
& \cdot \Psi_{r}\left(1, \overrightarrow{0}, I_{r}, \vec{u}\right) d \vec{u} d \varphi\left(u_{0}\right) .
\end{aligned}
$$

\section{Analytic Feynman Integrals and Conditional Analytic Feynman Integrals}

We begin this section with introducing the cylinder function on the analogue of Wiener space. Let $\left\{v_{1}, v_{2}, \ldots, v_{r}\right\}$ be an orthonormal subset of $L_{2}[0, T]$, let $r$ be any positive integer, let $1 \leq p \leq \infty$, and let $F$ be given by

$$
F(x)=f((\vec{v}, x))
$$

for $w_{\varphi}$ a.e. $x \in C[0, T]$, where $f \in L_{p}\left(\mathbb{R}^{r}\right)$. Without loss of generality we can take $f$ to be Borel measurable. In the following theorem we evaluate the Wiener and Feynman integrals of $F_{Z}$.

Theorem 11. Let $F$ be given by (37) with $1 \leq p \leq \infty$ and suppose that $\left\{M_{h} v_{1}, \ldots, M_{h} v_{r}\right\}$ is an independent subset of $L_{2}[0, T]$. Then for $\lambda \in \mathbb{C}_{+}$

$$
\begin{aligned}
E^{a n w_{\lambda}}\left[F_{Z}\right] & =\int_{\mathbb{R}^{r}} f(\vec{u}) \Psi_{r}\left(\lambda,(\vec{v}, a), \Sigma_{M_{h}}, \vec{u}\right) d \vec{u} \\
& =\int_{\mathbb{R}^{r}} f\left(\Sigma_{M_{h}}^{1 / 2} \vec{u}+(\vec{v}, a)\right) \Psi_{r}\left(\lambda, \overrightarrow{0}, I_{r}, \vec{u}\right) d \vec{u},
\end{aligned}
$$

where $\Sigma_{M_{h}}=\left[\left\langle M_{h} v_{i}, M_{h} v_{j}\right\rangle\right]_{r \times r}$ and $\Psi_{r}$ is given by (15). Moreover if $p=1$, then, for a nonzero real $q, E^{\text {anf }}{ }_{q}\left[F_{Z}\right]$ is given by the right hand side of (38) with replacing $\lambda$ by-iq.

Proof. Let $\lambda>0$. Replacing $h$ by $\lambda^{-1 / 2} h$ we have by Corollary 10

$$
\begin{aligned}
& \int_{C[0, T]} F_{Z}^{\lambda}(x) d w_{\varphi}(x) \\
& \quad=\int_{\mathbb{R}^{r}} f(\vec{u}) \Psi_{r}\left(\lambda,(\vec{v}, a), \Sigma_{M_{h}}, \vec{u}\right) d \vec{u} \\
& =\int_{\mathbb{R}^{r}} f\left(\Sigma_{M_{h}}^{1 / 2} \vec{u}+(\vec{v}, a)\right) \Psi_{r}\left(\lambda, \overrightarrow{0}, I_{r}, \vec{u}\right) d \vec{u} .
\end{aligned}
$$

For any real $N>0$

$$
\begin{aligned}
& \exp \left\{-\frac{N}{2}\left\langle\Sigma_{M_{h}}^{-1}(\vec{u}-(\vec{v}, a)), \vec{u}-(\vec{v}, a)\right\rangle_{\mathbb{R}}\right\} \\
& =\exp \left\{-\frac{N}{2}\left\|\Sigma_{M_{h}}^{-1 / 2}(\vec{u}-(\vec{v}, a))\right\|_{\mathbb{R}}^{2}\right\}
\end{aligned}
$$

which is integrable over $\vec{u}$, so that we have the theorem by the change of variable theorem, Morera's theorem, the uniqueness of analytic continuation, and the dominated convergence theorem.

Let $\left\{e_{11}, \ldots, e_{1 r}\right\}$ be the orthonormal set obtained from $\left\{M_{h} v_{1}, \ldots, M_{h} v_{r}\right\}$ by the Gram-Schmidt orthonormalization process. For $l=1, \ldots, r$ let $M_{h} v_{l}=\sum_{j=1}^{r} \alpha_{l j} e_{1 j}$ be the linear combination of $e_{1 j}$ 's and let $B_{1}=\left[\alpha_{l j}\right]_{r \times r}$ be the coefficient matrix of the combinations. Since

$$
\left\langle M_{h} v_{i}, M_{h} v_{j}\right\rangle=\sum_{l=1}^{r} \sum_{k=1}^{r}\left\langle\alpha_{i l} e_{1 l}, \alpha_{j k} e_{1 k}\right\rangle=\sum_{l=1}^{r} \alpha_{i l} \alpha_{j l}
$$

we have $\Sigma_{M_{h}}=B_{1} B_{1}^{T}$, where $B_{1}^{T}$ is the transpose of $B_{1}$.

We now have the following corollary by (41), Theorem 11, and the change of variable theorem.

Corollary 12. Under the assumptions as given in Theorem 11

$$
\begin{aligned}
E^{a n w_{\lambda}}\left[F_{Z}\right] & =\int_{\mathbb{R}^{r}} f(\vec{u}) \Psi_{r}\left(\lambda,(\vec{v}, a), B_{1} B_{1}^{T}, \vec{u}\right) d \vec{u} \\
& =\int_{\mathbb{R}^{r}} f\left(B_{1} \vec{u}+(\vec{v}, a)\right) \Psi_{r}\left(\lambda, \overrightarrow{0}, I_{r}, \vec{u}\right) d \vec{u} .
\end{aligned}
$$

Moreover if $p=1$, then $E^{a n f_{q}}\left[F_{Z}\right]$ is given by the right hand side of (42) with replacing $\lambda$ by-iq.

Theorem 13. Let $F$ be given by (37) with $1 \leq p \leq \infty$ and suppose that $\left\{\mathscr{P}^{\perp} M_{h} v_{1}, \ldots, \mathscr{P}^{\perp} M_{h} v_{r}\right\}$ is an independent subset of $L_{2}[0, T]$. Then for $\lambda \in \mathbb{C}_{+}$

$$
\begin{aligned}
& E^{a n w_{\lambda}}\left[F_{Z} \mid Z_{n+1}\right]\left(\vec{\xi}_{n+1}\right)=\int_{\mathbb{R}^{r}} f(\vec{u}) \\
& \cdot \Psi_{r}\left(\lambda,\left(\vec{v}, A+P_{b, n+1}\left(\vec{\xi}_{n+1}\right)\right), \Sigma_{\mathscr{P} \perp}, \vec{u}\right) d \vec{u} \\
& =\int_{\mathbb{R}^{r}} f\left(\Sigma_{\mathscr{P} \perp}^{1 / 2} \vec{u}+\left(\vec{v}, A+P_{b, n+1}\left(\vec{\xi}_{n+1}\right)\right)\right) \\
& \cdot \Psi_{r}\left(\lambda, \overrightarrow{0}, I_{r}, \vec{u}\right) d \vec{u}
\end{aligned}
$$

for a.e. $\vec{\xi}_{n+1} \in \mathbb{R}^{n+2}$, where $\Sigma_{\mathscr{P} \perp}=\left[\left\langle\mathscr{P}^{\perp} M_{h} v_{i}, \mathscr{P}^{\perp} M_{h} v_{j}\right\rangle\right]_{r \times r}$ and $A$ is given by (12). Moreover if $p=1$, then, for a nonzero real $q, E^{a n f_{q}}\left[F_{Z} \mid Z_{n+1}\right]$ is given by the right hand side of (43) with replacing $\lambda$ by $-i q$.

Proof. For $j=1, \ldots, r$ and $w_{\varphi}$ a.e. $x \in C[0, T]$ we have by Lemma 5

$$
\begin{aligned}
\left(v_{j}, X_{b, n+1}(x, \cdot)\right) & =\left(v_{j}, X(x, \cdot)-P_{b, n+1}(X(x, \cdot))\right) \\
& =\left(M_{h} v_{j}-\mathscr{P} M_{h} v_{j}, x\right) \\
& =\left(\mathscr{P}^{\perp} M_{h} v_{j}, x\right)
\end{aligned}
$$


so that for $\lambda>0$ and $\vec{\xi}_{n+1} \in \mathbb{R}^{n+2}$ we have by Corollary 10

$$
\begin{aligned}
& I_{F_{Z}}^{\lambda}\left(\vec{\xi}_{n+1}\right)=\int_{C[0, T]} F\left(\lambda^{-1 / 2} X_{b, n+1}(x, \cdot)+A\right. \\
& \left.\quad+P_{b, n+1}\left(\vec{\xi}_{n+1}\right)\right) d w_{\varphi}(x) \\
& \quad=\int_{C[0, T]} f\left(\lambda ^ { - 1 / 2 } \left(\left(\mathscr{P}^{\perp} M_{h} v_{1}, x\right), \ldots,\right.\right. \\
& \left.\quad\left(\mathscr{P}^{\perp} M_{h} v_{r}, x\right)\right)+(\vec{v}, A \\
& \left.\left.\quad+P_{b, n+1}\left(\vec{\xi}_{n+1}\right)\right)\right) d w_{\varphi}(x) \\
& \quad=\int_{\mathbb{R}^{r}} f(\vec{u}) \Psi_{r}\left(\lambda,\left(\vec{v}, A+P_{b, n+1}\left(\vec{\xi}_{n+1}\right)\right), \Sigma_{\mathscr{P} \perp},\right. \\
& \quad \vec{u}) d \vec{u}=\int_{\mathbb{R}^{r}} f\left(\Sigma_{\mathscr{P} \perp}^{1 / 2} \vec{u}+(\vec{v}, A\right. \\
& \left.\left.\quad+P_{b, n+1}\left(\vec{\xi}_{n+1}\right)\right)\right) \Psi_{r}\left(\lambda, \overrightarrow{0}, I_{r}, \vec{u}\right) d \vec{u},
\end{aligned}
$$

where $\Psi_{r}$ is given by (15). By the Morera and the dominated convergence theorem we have the theorem.

Let $\left\{e_{21}, \ldots, e_{2 r}\right\}$ be the orthonormal set obtained from $\left\{\mathscr{P}^{\perp} M_{h} v_{1}, \ldots, \mathscr{P}^{\perp} M_{h} v_{r}\right\}$ by the Gram-Schmidt orthonormalization process. For $l=1, \ldots, r$ let $\mathscr{P}^{\perp} M_{h} v_{l}=\sum_{j=1}^{r} \beta_{l j} e_{2 j}$ be the linear combination of $e_{2 j}$ 's and let $B_{2}=\left[\beta_{l j}\right]_{r \times r}$ be the coefficient matrix of the combinations.

We now have the following corollary by a similar calculation of (41).

Corollary 14. Under the assumptions as given in Theorem 13

$$
\begin{aligned}
& E^{a n w_{\lambda}}\left[F_{Z} \mid Z_{n+1}\right]\left(\vec{\xi}_{n+1}\right)=\int_{\mathbb{R}^{r}} f(\vec{u}) \\
& . \Psi_{r}\left(\lambda,\left(\vec{v}, A+P_{b, n+1}\left(\vec{\xi}_{n+1}\right)\right), B_{2} B_{2}^{T}, \vec{u}\right) d \vec{u} \\
& =\int_{\mathbb{R}^{r}} f\left(B_{2} \vec{u}+\left(\vec{v}, A+P_{b, n+1}\left(\vec{\xi}_{n+1}\right)\right)\right) \\
& . \Psi_{r}\left(\lambda, \overrightarrow{0}, I_{r}, \vec{u}\right) d \vec{u} .
\end{aligned}
$$

Moreover if $p=1$, then $E^{a n f_{q}}\left[F_{Z} \mid Z_{n+1}\right]$ is given by the right hand side of (46) with replacing $\lambda$ by-iq.

For $\vec{v}=\left(v_{1}, \ldots, v_{r}\right), \vec{u} \in \mathbb{R}^{r}, \lambda \in \mathbb{C}$, and any nonsingular positive $r \times r$ matrix $A_{r}$ on $\mathbb{R}$ let

$$
\begin{gathered}
\left\langle\vec{v} \alpha_{n+1}, \alpha_{n+1}\right\rangle=\left(\left\langle v_{1} \alpha_{n+1}, \alpha_{n+1}\right\rangle, \ldots,\left\langle v_{r} \alpha_{n+1}, \alpha_{n+1}\right\rangle\right), \\
\Lambda\left(A_{r}\right)=1+\left(b(T)-b\left(t_{n}\right)\right)\left\|A_{r}^{-1}\left\langle\vec{v} \alpha_{n+1}, \alpha_{n+1}\right\rangle\right\|_{\mathbb{R}}^{2}, \\
\Phi_{r}\left(\lambda, \vec{v}, A_{r}, \vec{u}\right)=\left[\frac{1}{\Lambda\left(A_{r}\right)}\right]^{1 / 2} \\
\cdot \exp \left\{\frac{\lambda}{2 \Lambda\left(A_{r}\right)}\left(b(T)-b\left(t_{n}\right)\right)\right. \\
\left.\cdot\left\langle\vec{u}, A_{r}^{-1}\left\langle\vec{v} \alpha_{n+1}, \alpha_{n+1}\right\rangle\right\rangle_{\mathbb{R}}^{2}\right\} .
\end{gathered}
$$

With the above notations, we have the following theorem.
Theorem 15. Let $F$ be given by (37) with $1 \leq p \leq \infty$. Then for $\lambda \in \mathbb{C}_{+}$

$$
\begin{aligned}
& E^{a n w_{\lambda}}\left[F_{Z} \mid Z_{n}\right]\left(\vec{\xi}_{n}\right)=\int_{\mathbb{R}^{r}} f\left(\Sigma_{\mathscr{P}^{\perp}}^{1 / 2} \vec{u}+(\vec{v}, A)\right. \\
& \left.+\left(\vec{v}, P_{b, n}\left(\vec{\xi}_{n}\right)\right)+\left(a(T)-a\left(t_{n}\right)\right)\left\langle\vec{v} \alpha_{n+1}, \alpha_{n+1}\right\rangle\right) \\
& . \Psi_{r}\left(\lambda, \overrightarrow{0}, I_{r}, \vec{u}\right) \Phi_{r}\left(\lambda, \vec{v}, \Sigma_{\mathscr{P}^{\perp}}^{1 / 2}, \vec{u}\right) d \vec{u}
\end{aligned}
$$

for a.e. $\vec{\xi}_{n} \in \mathbb{R}^{n+1}$, where $\left(\vec{v}, P_{b, n}\left(\vec{\xi}_{n}\right)\right)=\left(\left(v_{1}, P_{b, n}\left(\vec{\xi}_{n}\right)\right), \ldots\right.$, $\left.\left(v_{r}, P_{b, n}\left(\vec{\xi}_{n}\right)\right)\right)$. If $p=1$, then, for a nonzero real $q, E^{a n f_{q}}\left[F_{Z} \mid\right.$ $\left.Z_{n}\right]$ is given by the right hand side of the above equality with replacing $\lambda$ by-iq. Moreover the matrix $\Sigma_{\mathscr{P}^{\perp}}^{1 / 2}$ can be replaced by $B_{2}$ in the above results.

Proof. For $\vec{\xi}_{n}=\left(\xi_{0}, \xi_{1}, \ldots, \xi_{n}\right) \in \mathbb{R}^{n+1}$ let $\vec{\xi}_{n+1}=\left(\xi_{0}, \xi_{1}, \ldots\right.$, $\left.\xi_{n}, \xi_{n+1}\right)$, where $\xi_{n+1} \in \mathbb{R}$. For $\lambda>0$ we have by Lemma 6 , Theorem 13, and the change of variable theorem

$$
\begin{aligned}
K_{F_{Z}}^{\lambda} & \left(\vec{\xi}_{n}\right)=\int_{\mathbb{R}} \int_{\mathbb{R}^{r}} \Psi_{1}\left(\lambda, a(T)-a\left(t_{n}\right), b(T)\right. \\
& \left.-b\left(t_{n}\right), \xi_{n+1}-\xi_{n}\right) f\left(\Sigma_{\mathscr{P}^{\perp}}^{1 / 2} \vec{u}\right. \\
& \left.+\left(\vec{v}, A+P_{b, n+1}\left(\vec{\xi}_{n+1}\right)\right)\right) \\
& +\Psi_{r}\left(\lambda, \overrightarrow{0}, I_{r}, \vec{u}\right) d \vec{u} d \xi_{n+1} \\
& =\int_{\mathbb{R}^{r}} \int_{\mathbb{R}} \Psi_{1}\left(\lambda, a(T)-a\left(t_{n}\right), b(T)-b\left(t_{n}\right), \xi_{n+1}\right. \\
& \left.-\xi_{n}\right) f\left(\Sigma_{\mathscr{P} \perp}^{1 / 2} \vec{u}+(\vec{v}, A)+\left(\vec{v}, P_{b, n}\left(\vec{\xi}_{n}\right)\right)\right. \\
& \left.+\left(\xi_{n+1}-\xi_{n}\right)\left\langle\vec{v} \alpha_{n+1}, \alpha_{n+1}\right\rangle\right) \\
& +\Psi_{r}\left(\lambda, \overrightarrow{0}, I_{r}, \vec{u}\right) d \xi_{n+1} d \vec{u} \\
& =\int_{\mathbb{R}^{r}} \int_{\mathbb{R}} \Psi_{1}\left(\lambda, 0, b(T)-b\left(t_{n}\right), \xi_{n+1}\right) f\left(\Sigma_{\mathscr{P}^{\perp}}^{1 / 2} \vec{u}\right. \\
& +(\vec{v}, A)+\left(\vec{v}, P_{b, n}\left(\vec{\xi}_{n}\right)\right) \\
& +\left(a(T)-a\left(t_{n}\right)\right)\left\langle\vec{v} \alpha_{n+1}, \alpha_{n+1}\right\rangle \\
& \left.+\left\langle\vec{v} \alpha_{n+1}, \alpha_{n+1}\right\rangle \xi_{n+1}\right) \Psi_{r}\left(\lambda, \overrightarrow{0}, I_{r}, \vec{u}\right) d \xi_{n+1} d \vec{u} .
\end{aligned}
$$

Using the same method as used in the proof of Theorem 3.2 in [9]

$$
\begin{aligned}
& E^{a n w_{\lambda}}\left[F_{Z} \mid Z_{n}\right]\left(\vec{\xi}_{n}\right)=\int_{\mathbb{R}^{r}} f\left(\Sigma_{\mathscr{P}^{\perp}}^{1 / 2} \vec{u}+(\vec{v}, A)\right. \\
& \left.+\left(\vec{v}, P_{b, n}\left(\vec{\xi}_{n}\right)\right)+\left(a(T)-a\left(t_{n}\right)\right)\left\langle\vec{v} \alpha_{n+1}, \alpha_{n+1}\right\rangle\right) \\
& +\Psi_{r}\left(\lambda, \overrightarrow{0}, I_{r}, \vec{u}\right) \Phi_{r}\left(\lambda, \vec{v}, \Sigma_{\mathscr{P}^{\perp}}^{1 / 2}, \vec{u}\right) d \vec{u}
\end{aligned}
$$


by the Schwarz inequality and the Morera theorem. The final results follow from the dominated convergence theorem and by a similar calculation of (41).

Remark 16. (1) An orthonormal subset $\left\{v_{1}, v_{2}, \ldots, v_{r}\right\}$ of $L_{2}[0, T]$ such that both $\left\{M_{h} v_{1}, \ldots, M_{h} v_{r}\right\}$ and $\left\{\mathscr{P}^{\perp} M_{h} v_{1}, \ldots\right.$, $\left.\mathscr{P}^{\perp} M_{h} v_{r}\right\}$ are independent sets exists.

(2) It does not mean that $B_{1}=\Sigma_{M_{h}}^{1 / 2}$ in the equations of Corollary 12 and $B_{2}=\Sigma_{\mathscr{P} \perp}^{1 / 2}$ in the equations of Corollary 14 and of Theorem 15. They satisfy only the following equations:

$$
\begin{aligned}
& B_{1} B_{1}^{T}=\Sigma_{M_{h}}=\left(\Sigma_{M_{h}}^{1 / 2}\right)^{2}, \\
& B_{2} B_{2}^{T}=\Sigma_{\mathscr{P} \perp}=\left(\Sigma_{\mathscr{P} \perp}^{1 / 2}\right)^{2} .
\end{aligned}
$$

\section{Integral Transforms with Change of Scales}

In this section we derive change of scale transforms for the generalized conditional Wiener integrals of the function $F_{Z}$ which is introduced in the previous section. To derive these scale transforms we use multivariate normal distributions so that the orthonormalization process of projection vectors can be removed from the change of scale transforms in [8-10] and the transforms are expressed in terms of $F_{Z}$ itself.
For $\lambda \in \mathbb{C}$ and $x \in C[0, T]$ let

$$
\begin{aligned}
K_{1}(\lambda, x) & =\exp \left\{\frac{1-\lambda}{2}\left\|\Sigma_{M_{h}}^{-1 / 2}(\vec{v}, X(x, \cdot))\right\|_{\mathbb{R}}^{2}\right\} \\
& =\exp \left\{\frac{1-\lambda}{2}\left\|B_{1}^{-1}(\vec{v}, X(x, \cdot))\right\|_{\mathbb{R}}^{2}\right\} .
\end{aligned}
$$

We now have the following theorem.

Theorem 17. Let $1 \leq p \leq \infty$ and let $F$ be given by (37). Then for $\lambda \in \mathbb{C}_{+}$

$$
E^{a n w_{\lambda}}\left[F_{Z}\right]=\lambda^{r / 2} \int_{C[0, T]} K_{1}(\lambda, x) F_{Z}(x) d w_{\varphi}(x),
$$

where $K_{1}$ is given by (52). If $p=1$ and $q$ is a nonzero real number, then

$$
\begin{aligned}
& E^{a n f_{q}}\left[F_{Z}\right] \\
& \quad=\lim _{m \rightarrow \infty} \lambda_{m}^{r / 2} \int_{C[0, T]} K_{1}\left(\lambda_{m}, x\right) F_{Z}(x) d w_{\varphi}(x)
\end{aligned}
$$

for any sequence $\left\{\lambda_{m}\right\}_{m=1}^{\infty}$ in $\mathbb{C}_{+}$converging to $-i q$ as $m$ approaches to $\infty$.

Proof. For $\lambda \in \mathbb{C}_{+}$we have by (41), Corollary 10, and Theorem 11

$$
\begin{aligned}
\lambda^{r / 2} & \int_{C[0, T]} K_{1}(\lambda, x) F_{Z}(x) d w_{\varphi}(x)=\lambda^{r / 2} \int_{C[0, T]} f(\vec{v}, Z(x, \cdot)) \exp \left\{\frac{1-\lambda}{2}\left\langle\Sigma_{M_{h}}^{-1}(\vec{v}, X(x, \cdot)),(\vec{v}, X(x, \cdot))\right\rangle_{\mathbb{R}}\right\} d w_{\varphi}(x) \\
& =\lambda^{r / 2} \int_{C[0, T]} f(\vec{v}, Z(x, \cdot)) \exp \left\{\frac{1-\lambda}{2}\left\langle\Sigma_{M_{h}}^{-1}((\vec{v}, Z(x, \cdot))-(\vec{v}, a)),(\vec{v}, Z(x, \cdot))-(\vec{v}, a)\right\rangle_{\mathbb{R}}\right\} d w_{\varphi}(x) \\
& =\lambda^{r / 2} \int_{\mathbb{R}^{r}} f(\vec{u}) \Psi_{r}\left(1,(\vec{v}, a), \Sigma_{M_{h}}, \vec{u}\right) \exp \left\{\frac{1-\lambda}{2}\left\langle\Sigma_{M_{h}}^{-1}(\vec{u}-(\vec{v}, a)), \vec{u}-(\vec{v}, a)\right\rangle_{\mathbb{R}}\right\} d \vec{u} \\
& =\int_{\mathbb{R}^{r}} f(\vec{u}) \Psi_{r}\left(\lambda,(\vec{v}, a), \Sigma_{M_{h}}, \vec{u}\right) d \vec{u}=E^{a n w_{\lambda}}\left[F_{Z}\right],
\end{aligned}
$$

where $\Psi_{r}$ is given by (15). If $p=1$, the final result immediately follows from the dominated convergence theorem.

$$
\begin{aligned}
& \text { For } \lambda \in \mathbb{C}, x \in C[0, T] \text {, and } \vec{\xi}_{n+1} \in \mathbb{R}^{n+2} \text { let } \\
& \begin{array}{l}
K_{2}\left(\lambda, \vec{\xi}_{n+1}, x\right)=\left(\frac{1}{\left|\Sigma_{\mathscr{P} \perp}\right|}\right)^{1 / 2} \\
\cdot \exp \left\{-\frac{\lambda}{2}\left\|\Sigma_{\mathscr{P}^{\perp}}^{-1 / 2}\left(\vec{v}, Z(x, \cdot)-A-P_{b, n+1}\left(\vec{\xi}_{n+1}\right)\right)\right\|_{\mathbb{R}}^{2}\right\} \\
=\frac{1}{\left|B_{2}\right|} \\
\quad \cdot \exp \left\{-\frac{\lambda}{2}\left\|B_{2}^{-1}\left(\vec{v}, Z(x, \cdot)-A-P_{b, n+1}\left(\vec{\xi}_{n+1}\right)\right)\right\|_{\mathbb{R}}^{2}\right\} .
\end{array}
\end{aligned}
$$

We now have the following theorem.
Theorem 18. Let $1 \leq p \leq \infty$ and let $F$ be given by (37). Then for $\lambda \in \mathbb{C}_{+}$

$$
\begin{aligned}
& E^{a n w_{\lambda}}\left[F_{Z} \mid Z_{n+1}\right]\left(\vec{\xi}_{n+1}\right)=\left(\lambda^{r}\left|\Sigma_{M_{h}}\right|\right)^{1 / 2} \\
& \cdot \int_{C[0, T]} K_{1}(0, x) K_{2}\left(\lambda, \vec{\xi}_{n+1}, x\right) F_{Z}(x) d w_{\varphi}(x)
\end{aligned}
$$

for a.e. $\vec{\xi}_{n+1} \in \mathbb{R}^{n+2}$, where $K_{2}$ is given by (56). If $p=1$ and $q$ is a nonzero real number, then

$$
\begin{aligned}
& E^{a n f_{q}}\left[F_{Z} \mid Z_{n+1}\right]\left(\vec{\xi}_{n+1}\right)=\lim _{m \rightarrow \infty}\left(\lambda_{m}^{r}\left|\Sigma_{M_{h}}\right|\right)^{1 / 2} \\
& \cdot \int_{C[0, T]} K_{1}(0, x) K_{2}\left(\lambda_{m}, \vec{\xi}_{n+1}, x\right) F_{Z}(x) d w_{\varphi}(x)
\end{aligned}
$$


for any sequence $\left\{\lambda_{m}\right\}_{m=1}^{\infty}$ in $\mathbb{C}_{+}$converging to -iq as $m$ approaches to $\infty$. Moreover $\left|M_{h}\right|$ can be replaced by $\left|B_{1}\right|^{2}$ in the above equalities.

Proof. For $\lambda>0$ and a.e. $\vec{\xi}_{n+1} \in \mathbb{R}^{n+2}$ we have by Corollary 10

$$
\begin{aligned}
& \left(\lambda^{r}\left|\Sigma_{M_{h}}\right|\right)^{1 / 2} \int_{C[0, T]} K_{1}(0, x) K_{2}\left(\lambda, \vec{\xi}_{n+1}, x\right) F_{Z}(x) d w_{\varphi}(x)=\left(\frac{\lambda^{r}\left|\Sigma_{M_{h}}\right|}{\left|\Sigma_{\mathscr{P} \perp}\right|}\right)^{1 / 2} \int_{C[0, T]} f(\vec{v}, Z(x, \cdot)) \\
& \quad \cdot \exp \left\{\frac{1}{2}\left\langle\Sigma_{M_{h}}^{-1}((\vec{v}, Z(x, \cdot))-(\vec{v}, a)),(\vec{v}, Z(x, \cdot))-(\vec{v}, a)\right\rangle_{\mathbb{R}}\right. \\
& \left.\quad-\frac{\lambda}{2}\left\langle\Sigma_{\mathscr{P} \perp}^{-1}\left(\vec{v}, Z(x, \cdot)-A-P_{b, n+1}\left(\vec{\xi}_{n+1}\right)\right),\left(\vec{v}, Z(x, \cdot)-A-P_{b, n+1}\left(\vec{\xi}_{n+1}\right)\right)\right\rangle_{\mathbb{R}}\right\} d w_{\varphi}(x)=\left(\frac{\lambda^{r}\left|\Sigma_{M_{h}}\right|}{\left|\sum_{\mathscr{P} \perp}\right|}\right)^{1 / 2} \\
& \quad \cdot \int_{\mathbb{R}^{r}} f(\vec{u}) \Psi_{r}\left(1,(\vec{v}, a), \Sigma_{M_{h}}, \vec{u}\right) \exp \left\{\frac{1}{2}\left\langle\Sigma_{M_{h}}^{-1}(\vec{u}-(\vec{v}, a)), \vec{u}-(\vec{v}, a)\right\rangle_{\mathbb{R}}\right. \\
& \left.\quad-\frac{\lambda}{2}\left\langle\Sigma_{\mathscr{P} \perp}^{-1}\left(\vec{u}-\left(\vec{v}, A+P_{b, n+1}\left(\vec{\xi} \xi_{n+1}\right)\right)\right), \vec{u}-\left(\vec{v}, A+P_{b, n+1}\left(\overrightarrow{\xi_{n+1}}\right)\right)\right\rangle_{\mathbb{R}}\right\} d \vec{u}=\int_{\mathbb{R}^{r}} f(\vec{u}) \Psi_{r}\left(\lambda,\left(\vec{v}, A+P_{b, n+1}\left(\vec{\xi}_{n+1}\right)\right),\right. \\
& \left.\quad \sum_{\mathscr{P} \perp}, \vec{u}\right) d \vec{u} .
\end{aligned}
$$

By (41), the analytic continuation, the dominated convergence theorem, and Theorem 13 we have the theorem.

By Theorems 15 and 18 we have the final theorem.

Theorem 19. Let $1 \leq p \leq \infty$ and let $F$ be given by (37). Then for $\lambda \in \mathbb{C}_{+}$

$$
\begin{aligned}
& E^{a n w_{\lambda}}\left[F_{Z} \mid Z_{n}\right]\left(\vec{\xi}_{n}\right)=\left(\lambda^{r}\left|\Sigma_{M_{h}}\right|\right)^{1 / 2} \\
& \cdot \int_{\mathbb{R}} \int_{C[0, T]} \Psi_{1}\left(\lambda, a(T)-a\left(t_{n}\right), b(T)\right. \\
& \left.\quad-b\left(t_{n}\right), \xi_{n+1}-\xi_{n}\right) K_{1}(0, x) K_{2}\left(\lambda, \vec{\xi}_{n+1}, x\right) \\
& \cdot F_{Z}(x) d w_{\varphi}(x) d \xi_{n+1}
\end{aligned}
$$

for a.e. $\vec{\xi}_{n}=\left(\xi_{0}, \xi_{1}, \ldots, \xi_{n}\right) \in \mathbb{R}^{n+1}$, where $\vec{\xi}_{n+1}=\left(\xi_{0}, \xi_{1}, \ldots\right.$, $\left.\xi_{n}, \xi_{n+1}\right)$ and $\Psi_{1}, K_{2}$ are given by (15) and (56), respectively. If $p=1$ and $q$ is a nonzero real number, then

$$
\begin{aligned}
& E^{a n f_{q}}\left[F_{Z} \mid Z_{n}\right]\left(\vec{\xi}_{n}\right)=\lim _{m \rightarrow \infty}\left(\lambda_{m}^{r}\left|\Sigma_{M_{h}}\right|\right)^{1 / 2} \\
& \cdot \int_{\mathbb{R}} \int_{C[0, T]} \Psi_{1}\left(\lambda_{m}, a(T)-a\left(t_{n}\right), b(T)\right. \\
& \left.-b\left(t_{n}\right), \xi_{n+1}-\xi_{n}\right) K_{1}(0, x) K_{2}\left(\lambda_{m}, \vec{\xi}_{n+1}, x\right) \\
& \cdot F_{Z}(x) d w_{\varphi}(x) d \xi_{n+1}
\end{aligned}
$$

for any sequence $\left\{\lambda_{m}\right\}_{m=1}^{\infty}$ in $\mathbb{C}_{+}$converging to -iq as $m$ approaches to $\infty$. Moreover $\left|M_{h}\right|$ can be replaced by $\left|B_{1}\right|^{2}$ in the above equalities.
Remark 20. (1) Letting $\lambda=\rho^{-2}$ in the theorems of this section, where $\rho>0$, we have the change of scale formulas for $E\left[F_{Z}(\rho \cdot)\right], E\left[F_{Z}(\rho \cdot) \mid Z_{n}(\rho \cdot)\right]$, and $E\left[F_{Z}(\rho \cdot) \mid Z_{n+1}(\rho \cdot)\right]$ as integral transforms.

(2) If $a=0$ and $h=1$ a.e., then we can obtain Theorems 5.1 and 5.2 in [9].

(3) If $a=0, h=1$ a.e., and $\varphi=\delta_{0}$ which is the Dirac measure concentrated at 0 , then we can obtain the change of scale transforms in [10].

(4) The results in this paper are independent of a particular choice of the initial distribution $\varphi$.

\section{Competing Interests}

The author declares that they have no competing interests.

\section{Acknowledgments}

This research was supported by Basic Science Research Program through the National Research Foundation (NRF) of Korea funded by the Ministry of Education (2013R1A1A2058991).

\section{References}

[1] R. H. Cameron, "The translation pathology of Wiener space," Duke Mathematical Journal, vol. 21, pp. 623-627, 1954.

[2] R. H. Cameron and W. T. Martin, "The behavior of measure and measurability under change of scale in Wiener space," Bulletin of the American Mathematical Society, vol. 53, pp. 130-137, 1947.

[3] R. H. Cameron and D. A. Storvick, "Change of scale formulas for Wiener integral," Rendiconti del Circolo Matematico di Palermo, no. 2, supplement 17, pp. 105-115, 1987. 
[4] B. S. Kim, "Relationship between the Wiener integral and the analytic Feynman integral of cylinder function," Journal of the Chungcheong Mathematical Society, vol. 27, no. 2, pp. 249-260, 2014.

[5] I. Yoo and D. Skoug, "A change of scale formula for Wiener integrals on abstract Wiener spaces," International Journal of Mathematics and Mathematical Sciences, vol. 17, no. 2, pp. 239247, 1994.

[6] I. Yoo and D. Skoug, "A change of scale formula for Wiener integrals on abstract Wiener spaces II," Journal of the Korean Mathematical Society, vol. 31, no. 1, pp. 115-129, 1994.

[7] I. Yoo, T. S. Song, B. S. Kim, and K. S. Chang, "A change of scale formula for Wiener integrals of unbounded functions," The Rocky Mountain Journal of Mathematics, vol. 34, no. 1, pp. 371-389, 2004.

[8] D. H. Cho, "Change of scale formulas for conditional Wiener integrals as integral transforms over Wiener paths in abstract Wiener space," Communications of Korean Mathematical Society, vol. 22, no. 1, pp. 91-109, 2007.

[9] D. H. Cho, B. J. Kim, and I. Yoo, "Analogues of conditional Wiener integrals and their change of scale transformations on a function space," Journal of Mathematical Analysis and Applications, vol. 359, no. 2, pp. 421-438, 2009.

[10] I. Yoo, K. S. Chang, D. H. Cho, B. Kim, and T. S. Song, "A change of scale formula for conditional Wiener integrals on classical Wiener space," Journal of the Korean Mathematical Society, vol. 44, no. 4, pp. 1025-1050, 2007.

[11] M. K. Im and K. S. Ryu, "An analogue of Wiener measure and its applications," Journal of the Korean Mathematical Society, vol. 39, no. 5, pp. 801-819, 2002.

[12] D. H. Cho, "Analogues of conditional Wiener integrals with drift and initial distribution on a function space," Abstract and Applied Analysis, vol. 2014, Article ID 916423, 12 pages, 2014.

[13] D. H. Cho, "Evaluation formulas for generalized Wiener integrals with drift and initial distribution," International Journal of Mathematical Analysis, vol. 8, no. 1-4, pp. 51-65, 2014.

[14] I. Pierce, On a family of generalized Wiener spaces and applications [Ph.D. thesis], University of Nebraska-Lincoln, Lincoln, Neb, USA, 2011.

[15] D. M. Chung, C. Park, and D. Skoug, "Generalized Feynman integrals via conditional Feynman integrals," The Michigan Mathematical Journal, vol. 40, no. 2, pp. 377-391, 1993. 


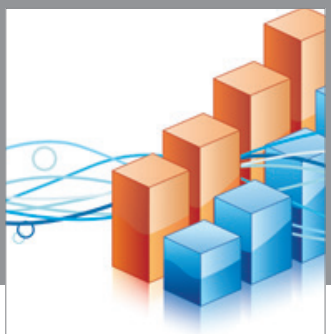

Advances in

Operations Research

vatem alat4

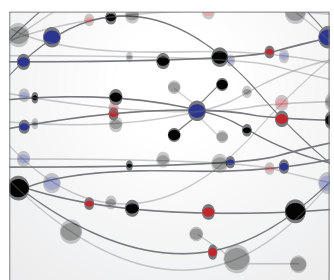

\section{The Scientific} World Journal
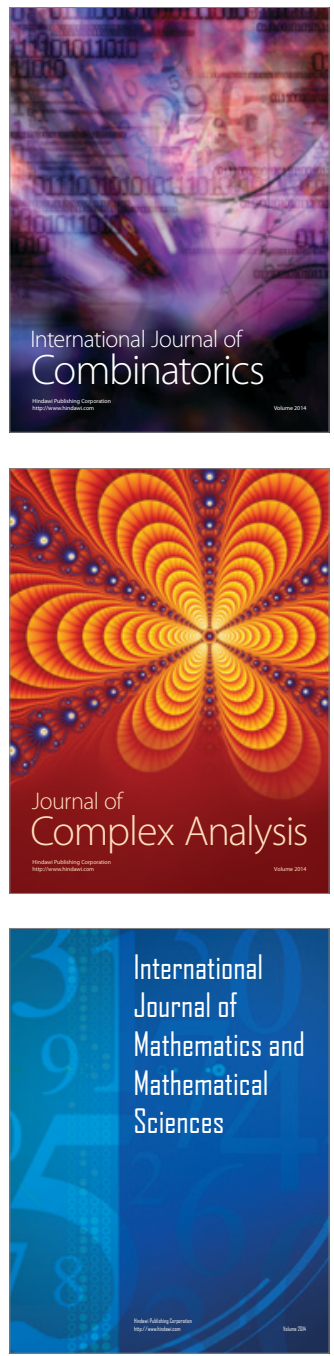
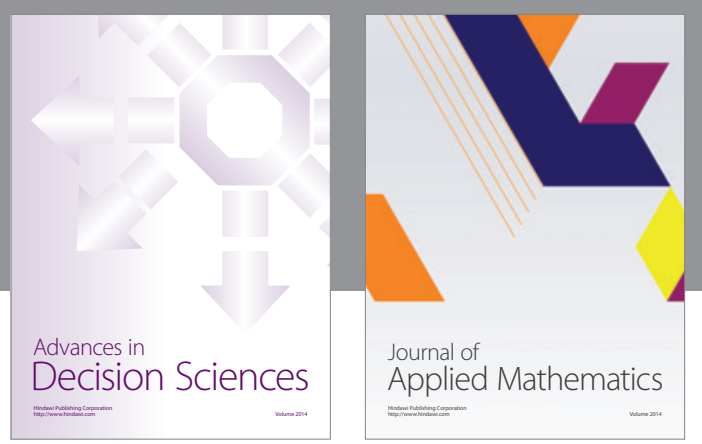

Algebra

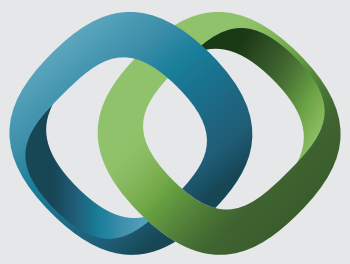

\section{Hindawi}

Submit your manuscripts at

http://www.hindawi.com
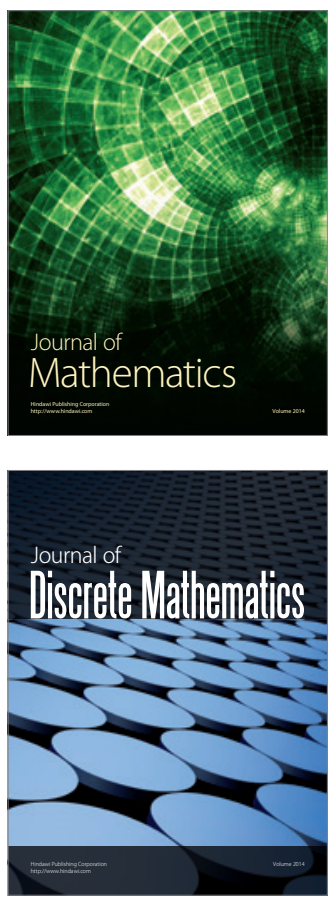

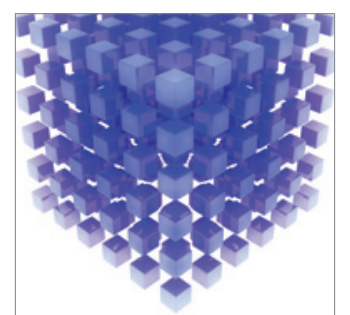

Mathematical Problems in Engineering
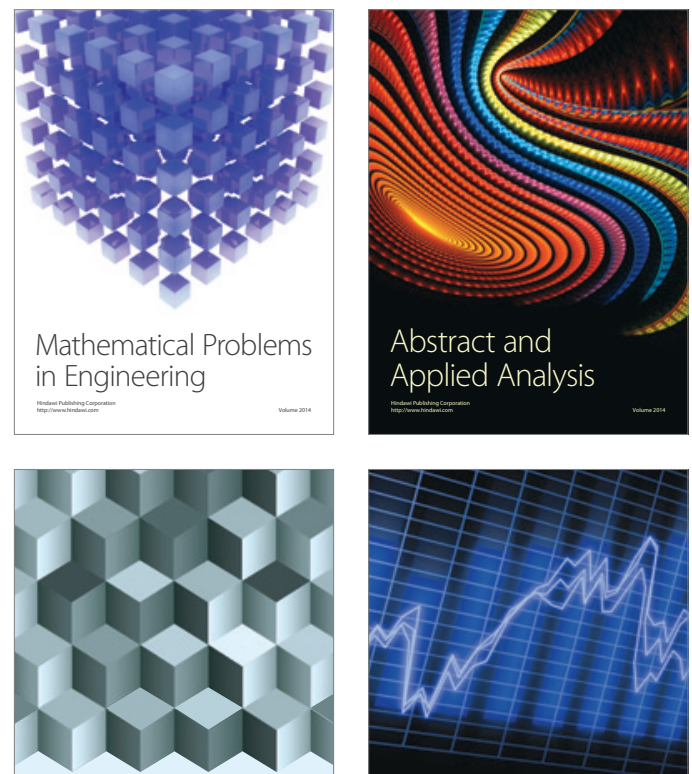

Journal of

Function Spaces

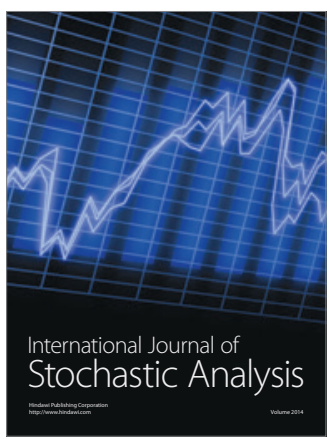

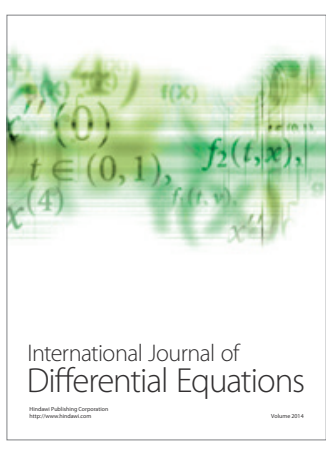
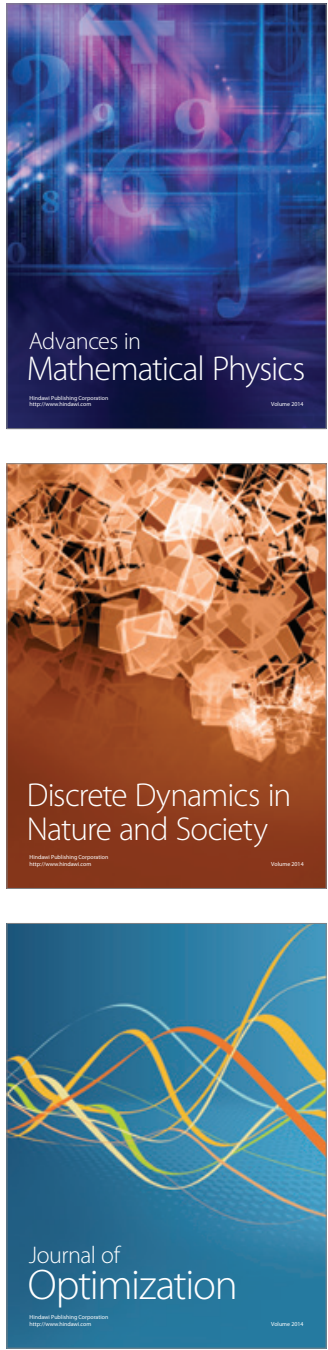